\title{
Cyanidin-3-glucoside Alleviates 4-Hydroxyhexenal-Induced NLRP3 Inflammasome Activation via JNK-c-Jun/AP-1 Pathway in Human Retinal Pigment Epithelial Cells
}

\author{
Xiaolu Jin $\mathbb{D}^{1},{ }^{1}$ Chengtao Wang, ${ }^{2}$ Wei Wu, ${ }^{3}$ Tingting Liu, ${ }^{4}$ Baoping Ji $\mathbb{D}^{1},{ }^{1}$ and Feng Zhou $\mathbb{D}^{1}$ \\ ${ }^{1}$ Beijing Advanced Innovation Center for Food Nutrition and Human Health, College of Food Science and Nutritional Engineering, \\ China Agricultural University, Beijing 100083, China \\ ${ }^{2}$ Beijing Engineering and Technology Research Center of Food Additives, Beijing Technology \& Business University, \\ Beijing 100048, China \\ ${ }^{3}$ College of Engineering, China Agricultural University, Beijing 100083, China \\ ${ }^{4}$ Key Laboratory of Agricultural Big Data, Ministry of Agriculture, Beijing 100081, China
}

Correspondence should be addressed to Baoping Ji; jbp@cau.edu.cn and Feng Zhou; zf@cau.edu.cn

Received 24 February 2018; Accepted 8 April 2018; Published 30 April 2018

Academic Editor: Bill B. Chen

Copyright (C) 2018 Xiaolu Jin et al. This is an open access article distributed under the Creative Commons Attribution License, which permits unrestricted use, distribution, and reproduction in any medium, provided the original work is properly cited.

\begin{abstract}
Recently, the NLRP3 inflammasome activation in the eyes has been known to be associated with the pathogenesis of age-related macular degeneration. The aim of this study was to investigate the protective effects of cyanidin-3-glucoside (C3G), an important anthocyanin with great potential for preventing eye diseases, against 4-hydroxyhexenal- (HHE-) induced inflammatory damages in human retinal pigment epithelial cells, ARPE-19. We noticed that C3G pretreatment to the ARPE-19 cells rescued HHE-induced antiproliferative effects. Cell apoptosis ratio induced by HHE was also decreased by C3G, measured by flow cytometry. The activation of NLRP3 inflammasome induced by HHE was found with increases of caspase- 1 activity, proinflammatory cytokine releases (IL-1 $\beta$ and IL-18), and NLRP3 inflammasome-related gene expressions (NLRP3, IL-1 $\beta$, IL-18, and caspase-1). The C3G showed potent inhibitive effects on these NLRP3 inflammasome activation hallmarks induced by HHE. Moreover, we noticed that the C3G's pretreatment leads to a delayed and a decreased JNK activation in HHE-challenged ARPE-19 cells. Finally, using a luciferase reporter gene assay system, we demonstrated that HHE-induced activation protein- (AP-) 1 transcription activity was abolished by C3G pretreatment in a dose-dependent manner. Taken together, these data showed that HHE leads to inflammatory damages to ARPE-19 cells while C3G has great protective effects, highlighting future potential applications of C3G against AMD-associated inflammation.
\end{abstract}

\section{Introduction}

Currently, age-related macular degeneration (AMD) is becoming the leading cause of elderly legal blindness in the world [1]. The Bruch's membrane, choroid, photoreceptors, and retinal pigment epithelium (RPE) are primarily affected by $\mathrm{AMD}$, characterized with morphological and functional abnormalities. During AMD, the RPE becomes progressively dysfunctional and eventually degenerates, which leads to the death of photoreceptors and finally causes the loss of the visual function. The etiology and the molecular pathogenesis of AMD remain unclear, particularly for the atrophic subtype of this disease. Experimental and clinical studies recently identified that the intense parainflammation to the RPE is a contributing factor in the development of AMD $[2,3]$. Therefore, recent studies have focused on the inflammasome signaling pathway, which leads to the development of clinically relevant novel therapeutic strategies $[4,5]$.

Inflammation is involved in innate immune response against exogenous pathogens and is considered as the first line of defense against pathogenic microbes as well as cellular stress [6]. The activation of inflammasome is important for transducing these "danger" signals for triggering inflammation. Inflammasomes lead to the activation of caspase- 1 and 
the following cleavage of IL- $1 \beta$ and IL-18, the two major proinflammatory cytokines. A series of inflammasome complexes have been clarified, and each of them was identified with a unique pattern recognition receptor (PRR) and activation triggers. Among these complexes, NLRP3 inflammasome is the best characterized one, which is consisted by NLRP3, apoptosis-associated speck-like protein, serinethreonine kinase NEK7, and procaspase-1 [7]. The NLRP3 inflammasome is activated in a 2 -step process. First, NF- $\kappa \mathrm{B}$ signaling is induced through pathogen-associated molecular patterns (PAMPs) or danger-associated molecular pattern(DAMP-) mediated activation of TLR4 or TNFR, which result in the increased expression of NLRP3, pro-IL-1 $\beta$, and pro-IL-18. Next, inflammatory microenvironmental factors (whole pathogens, PAMPs/DAMPs, or endogenous factors) induced an indirect activation of NLRP3 and induced the activation of caspase- 1 which eventually leads to the maturation and secretion of IL- $1 \beta$ and IL-18 [8]. The NLRP3 inflammasome has been found to be present in samples from AMD patients [9]. Several compounds associated with AMD have been shown to activate the NLRP3 inflammasome, like the complement component C5a and AMD including drusen components including $\mathrm{C} 1 \mathrm{q}$ and amyloid- $\beta$ [3], the lipofuscin component $\mathrm{N}$-retinylidene- $\mathrm{N}$-retinyl-ethanolamine (A2E), and the lipid peroxidation product 4-hydroxynonenal (HNE) [10]. Previous studies also noticed that photooxidative damage to the RPE-accumulated lipofuscin will activate the NLRP3 inflammasome [11].

Current thinking suggests that RPE cells are constantly exposed to lipid peroxidation. In the retinal tissues, free radicals can directly attack critical biomolecules including polyunsaturated fatty acids (PUFAs) and lead to its degradation into oxidized products, including aldehydes. Among these hazardous lipid products, 4-hydroxynonenal (4-HNE) and 4-hydroxyhexenal (4-HHE) are the most investigated unsaturated aldehydes. 4-HNE is derived from n-6 PUFAs, like linoleic acid and arachidonic acid, while 4-HHE is formed from n-3 PUFAs such as docosahexaenoic acid (DHA), eicosapentaenoic acid (EPA), and linolenic acid [12]. These three major unsaturated fatty acids reached about $50 \%, 10 \%$, and $8 \%$ of the total fatty acids, respectively, in human retina [13]. Due to the structure similarities of 4HNE and 4-HHE, these two aldehydes showed a number of cytotoxic effects, including inhibition on selective enzyme activity, induction on cell cycle arrest, and cell apoptosis [14]. Recent study also highlighted the roles of 4-HNE during NLRP3 inflammasome activation in human retinal pigment epithelium cells, ARPE-19 [11]. In this work, the potential damages caused by daily visible light exposure on retinal UFAs were evaluated via a simulated in vitro model [13]. Moreover, our previous study showed that the lipid peroxidation of DHA affects the physiological health of the retina cells $[13,15]$. Nevertheless, as the major lipid oxidized products from DHA, whether 4-HHE has a proinflammatory effect is still unknown.

Anthocyanins are strong antioxidants which have been evidenced to be beneficial for vision [16]. Cyanidin-3glucoside $(\mathrm{C} 3 \mathrm{G})$ is an important anthocyanin found in purple fruits and rice with great beneficial potentials for preventing eye diseases $[17,18]$. It has been examined with respect to different steps in the visual signal transduction process. Previous studies noticed that it inhibits the photooxidation of RPE cells via facilitating the regeneration of rhodopsin in rod photoreceptors $[19,20]$. Moreover, C3G has been reported to regulate the visual signal transduction. For example, in rod outer segments, C3G inhibited the activation of the G-protein induced by light exposure via metarhodopsin II [21]. Our previous studies confirmed that the retinal protective activity of $\mathrm{C} 3 \mathrm{G}$ against light-induced retinal injury was confirmed in vivo while the underlying mechanisms remain unclear $[13,18,22]$. In this article, we aim to know whether 4-HHE might induce activation of inflammasome signaling in ARPE-19 cells and furthermore that the polyphenol compound, C3G, is able to protect RPE cells against inflammatory damage.

\section{Material and Methods}

2.1. Cell Culture and Treatment. Human retinal pigment epithelium cells, ARPE-19, were obtained from the ATCC and cultured in DMEM/F12 medium (Gibco BRL, Grand Island, NY) with $10 \%$ fetal bovine serum (FBS; Thermo Fisher Scientific, Waltham, MA) in a humidified incubator at $37^{\circ} \mathrm{C}$ in $5 \%$ $\mathrm{CO}_{2}$, supplemented with $100 \mathrm{U} / \mathrm{mL}$ penicillin and $100 \mu \mathrm{g} / \mathrm{mL}$ streptomycin (Sigma-Aldrich, St. Louis, MO, USA). For cell treatment, cells were washed with prewarmed PBS then the complete culture medium was replaced by FBS-free medium. To induce inflammatory damage, 4-hydroxyhexenal (Cayman Chemical, Ann Arbor, MI, USA) which were dissolved in ethanol were challenged to the cells for $24 \mathrm{~h}$. After determining the optimal dosage of cyanidin-3-glucoside (Yuanye Biological Technology Co. Ltd., Shanghai, China), which was dissolved in DMSO, cells were pretreated with C3G for $2 \mathrm{~h}$ before 4-HHE stimulations as indicated in the experiments. All studies were performed using $70-80 \%$ confluent cells before treatment.

2.2. Cell Viability Assays. To measure the cytotoxicity of HNE and $\mathrm{C} 3 \mathrm{G}$ treatments, cell medium samples were analyzed for CCK-8 and lactate dehydrogenase (LDH) enzyme activity. After designed treatments, $10 \mu \mathrm{L}$ of CCK- 8 solution (Dojindo, Kumamoto, Japan) was added into 96-well plates and $\mathrm{OD} 450 \mathrm{~nm}$ was measured using a microplate reader (M5, MD, USA). The LDH activity was also determined by a commercial kit (Beyotime, Haimen, China) according to the manufacturer's instructions.

2.3. Cell Apoptosis Measurement. ARPE-19 cell apoptosis was assessed using an Annexin V-FITC Apoptosis Detection Kit (BD Biosciences Pharmingen, San Diego, CA, USA). Briefly, after indicated treatments, cells were washed and harvested then resuspended in $100 \mu \mathrm{L}$ Annexin Binding Buffer. Cells were stained with Annexin V-FITC $(5 \mu \mathrm{L})$ and propidium iodide (PI, $1 \mu \mathrm{L}$ ) solution. The flow cytometric analysis was performed using a FACScan flow cytometer (Becton Dickinson, Franklin Lakes, NJ, USA).

Cellular caspase- 1 activity was determined using a commercial kit based on colorimetric assay according to the 
manufacturer's instructions (Beyotime, Haimen, China). Briefly, cells were treated as designed then lysed and centrifuged at $10,000 \times \mathrm{g}$ for $1 \mathrm{~min}$ at room temperature. Total cytosolic protein $(50 \mu \mathrm{g})$ was incubated with acetyl-TyrVal-Ala-Asp p-nitroaniline (Ac-YVAD-pNA, 20 nM) for $2 \mathrm{~h}$ at $37^{\circ} \mathrm{C}$. The absorbance values were measured at $405 \mathrm{~nm}$ using a microplate reader (M5, MD, USA).

2.4. Enzyme-Linked Immunosorbent Assay (ELISA). Cell culture supernatants from treated cells were collected, and cytokines were measured by using commercial IL-1 $\beta$ and IL-18 ELISA kits (R\&D Systems, Minneapolis, MN, USA), following the manufacturer's instructions.

2.5. Quantitative Real-Time PCR. According to the instructions from the manufacturer, cellular RNA was extracted using the RNeasy ${ }^{\circledR}$ Plus Mini Kit (Qiagen, Valencia, CA, USA). Reverse transcription was carried out using the PrimeScript RT Reagent Kit (TaKaRa, Dalian, China). Real-time PCR was performed with the $7500 \mathrm{c}$ Real-time PCR Detection System (Applied Biosystems, Carlsbad, CA, USA) with SYBR Premix Ex Taq (TaKaRa) following the manufacturer's instructions. Primers were designed to flank introns with the Primer 5 software (Premier Biosoft, Palo Alto, CA, USA), and the primers sets were as follows: GAPDH, $5^{\prime}$-AGGGAT GATGTTCTGGAGAG-3' (F) and $5^{\prime}$-TCAAGATCATC AGCAATGCC-3' (R); NLRP3, $5^{\prime}$-TCGGAGATTGTGGT TGGG-3 ${ }^{\prime}(\mathrm{F})$ and $5^{\prime}$-GGGCGTTGTCACTCAGGT- $3^{\prime}$ (R); $I L-1 \beta, 5^{\prime}$-CTAAACAGATGAAGTGCTCCTTCC- $3^{\prime}(\mathrm{F})$ and $5^{\prime}$-CACATAAGCCTCGTTATCCCA-3' $(\mathrm{R}) ; \quad I L-18,5^{\prime}$-AT CAGGATCCTTTGGCAAGCTTGAATCTAAATTATC- $3^{\prime}$ (F) and $5^{\prime}$-ATAGGTCGACTTCGTTTTGAACAGTGAAC ATTATAG-3' (R) [23]; and caspase-1, 5' ${ }^{\prime}$-TGGTCTTGT GACTTGGAGGA- $3^{\prime}$ (F) and $5^{\prime}$-TGGCTTCTTATTGGC ACGAT-3 $^{\prime}$ (R) [24]. Data were calculated using equation $2^{-\Delta \Delta \mathrm{Ct}}$ and normalized to the expression of the housekeeping gene (GAPDH) and expressed as fold change against the control group.

2.6. Western Blot Analysis. Cellular proteins were lysed, and equal amounts of protein $(20 \mu \mathrm{g})$ were separated by SDSPAGE and then transferred to PVDF membranes, which were incubated with primary antibodies, phospho-JNK1/2 (Thr183/Tyr185) and $\beta$-tubulin (Cell Signaling Technology, Danvers, MA; Abcam, Cambridge, UK). Bound antibody complexes were visualized using NBT/BCIP solution with color development buffer.

2.7. Luciferase Reporter Assay. ARPE-19 cells were seeded in $6 \mathrm{~cm}$ plates and transfected with $3 \mu \mathrm{g}$ of pGL3-AP1 vector. After transfection, cells were separated into a 24 -well plate followed by treatment with the indicated concentrations of C3G for $2 \mathrm{~h}$ before the addition of HHE for another $24 \mathrm{~h}$. Luciferase activity was measured using the dual luciferase assay system (Promega, Madison, WI, USA) according to the manufacturer's instructions. A Renilla luciferaseexpressing vector (pRL-TK) was cotransfected with firefly luciferase and used as the internal control. Promoter activity is presented as multiples of the control.

2.8. Statistical Analyses. Quantitative data are presented as the arithmetic mean \pm standard error of the mean (SEM) for each treatment group. The effect of treatments was determined by one-way analysis of variance (ANOVA), and differences between treatments were analyzed post hoc by Tukey's honest significant difference test. A $P$ value less than 0.05 was considered statistically significant. All statistical tests were performed using SPSS version 17.0 (SPSS Inc., Chicago, IL).

\section{Results}

3.1. C3G Inhibited HHE-Induced Antiproliferative Effect via Suppressing RPE Cell Apoptosis. As shown in Figure 1(a), various concentrations of HHE (10 to $200 \mu \mathrm{M}$ ) were applied to the ARPR-19 cells and we noticed notable antiproliferative effects followed by a $24 \mathrm{~h}$ HHE treatment, with significant decreased of reduction in the viability, and dramatical LDH enzyme releases $(25,50,100$, and $200 \mu \mathrm{M} ; P<0.05$ and $P<0.01$, resp.). Moreover, to explore whether $\mathrm{C} 3 \mathrm{G}$ has any protective effects against HHE-induced antiproliferative effect, we tested various concentrations of $\mathrm{C} 3 \mathrm{G}$ and pretreated them to the cells $2 \mathrm{~h}$ before the HHE challenge. As shown in Figure 1(b), when C3Gs $(25,50$, and $100 \mu \mathrm{M})$ were added to the cell cultures, the compromised metabolic activity of cells by HHE were improved. In addition, HHEinduced LDH releases were significantly diminished by C3G. On a molecular level, we noticed that cell apoptosis ratio induced by HHE was decreased by $\mathrm{C} 3 \mathrm{G}$, measured by flow cytometry (Figure 1(c)). Furthermore, caspase-1 activity was increased by 2.03 -fold in ARPE- 19 cells after $24 \mathrm{~h}$ HHE treatment, and 50 and $100 \mu \mathrm{M} \mathrm{C} 3 \mathrm{G}$ treatment to the HHE-challenged cells reduced the caspase-1 activity significantly (Figure $1(\mathrm{~d})$ ).

3.2. C3G Reduced the Production of IL-1 $\beta$ and IL-18 Induced by HHE in RPE Cells. We hypothesized that HHE might lead to inflammatory damages in ARPE-19 cells and C3G might exert an anti-inflammatory effect. Therefore, we next tested proinflammatory cytokine releases in HHE- and C3Gtreated ARPE-19 cells. As shown in Figure 2, $50 \mu \mathrm{M}$ HHE for $24 \mathrm{~h}$ leads to dramatical increases of IL- $1 \beta$ and IL- 18 . Strikingly, compared with the HHE-treated control, C3G $(100 \mu \mathrm{M})$ pretreatment to ARPE-19 cells for $2 \mathrm{~h}$ leads to a decrease of IL- $1 \beta$ and IL- 18 for $66 \%$ and $45 \%$, respectively.

3.3. C3G Inhibited the Activation of NLRP3 Inflammasome Induced by HHE in RPE Cells. To evaluate the roles of the NLRP3 inflammasome complex during C3G-induced protective effects, ARPE-19 cells were pretreated with C3G and challenged with HHE. Several key NLRP3 inflammasome components, including NLRP3, IL-1 $\beta$, IL-18, and caspase-1 mRNA levels, are measured by qRT-PCR. As shown in Figure 3(a), C3G significantly decreased the HHE-induced mRNA level of NLRP3 in ARPE-19 cells. Activation of NLRP3 inflammasome was also notified by upregulations of IL-1 $\beta$, IL-18, and caspase-1. C3G showed potent inhibitive 


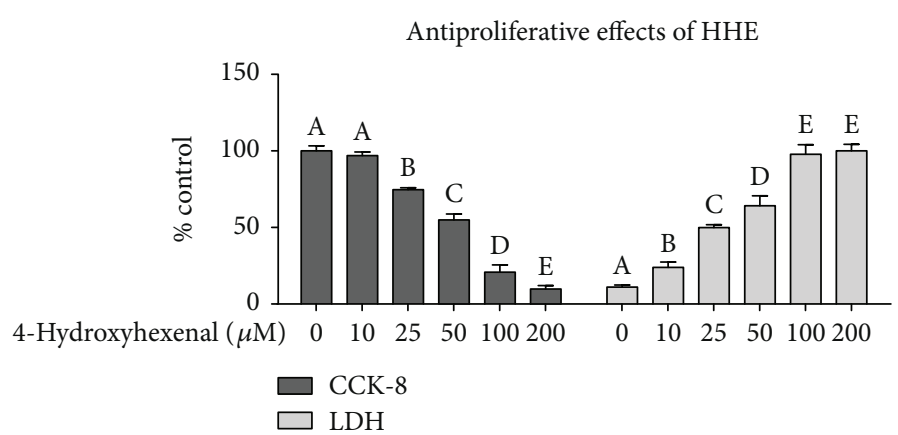

(a)

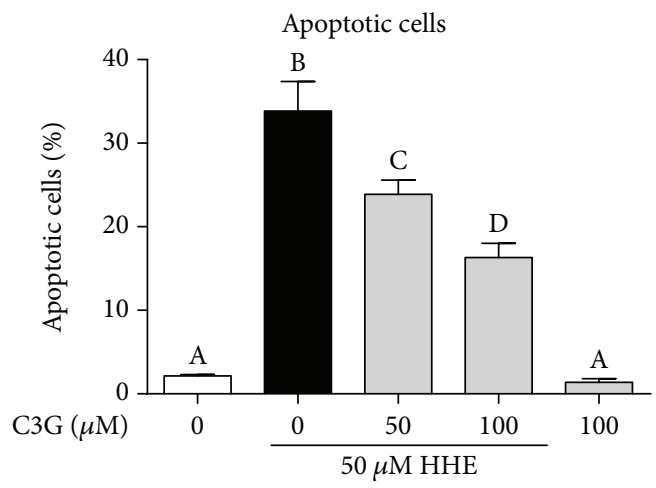

(c)

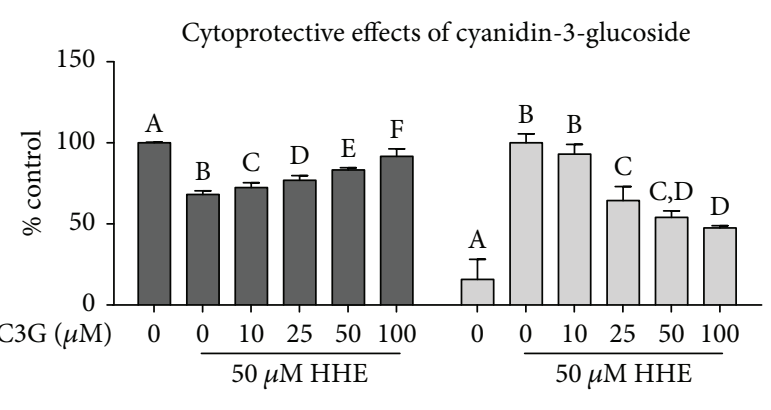

CCK-8

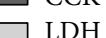

(b)

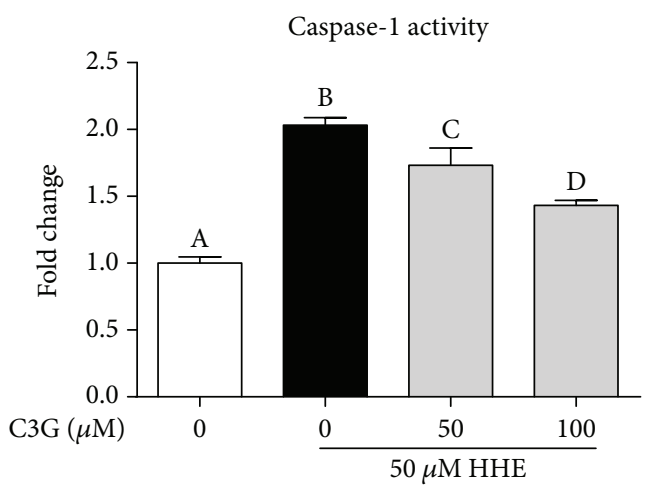

(d)

FIGURE 1: C3G inhibited HHE-induced antiproliferative effect via suppressing RPE cell apoptosis. (a) HHE showed antiproliferative effects in ARPE-19 cells. ARPE-19 cells were incubated with various dosages of HHE for $24 \mathrm{~h}$ in serum-free culture mediums, and then the cell viability was measured by cell counting kit- (CCK-) 8 and LDH (lactate dehydrogenase) tests as described in Materials and Methods. (b) C3G showed protective effects against HHE-induced antiproliferative effects in ARPE-19 cells. Cells are incubated with or without C3G for $2 \mathrm{~h}$ then challenged with HHE for $24 \mathrm{~h}$ in serum-free culture mediums. The cell viability was measured by CCK-8 assay and LDH tests. (c) Cell apoptosis induced by HHE was inhibited by C3G. C3Gs $(50,100 \mu \mathrm{M})$ were pretreated to the ARPE-19 cells for $2 \mathrm{~h}$, and then HHE was challenged for $24 \mathrm{~h}$ in serum-free culture mediums. ARPE-19 cell apoptosis was determined by Annexin V/PI staining. Data reported as percentage of Annexin V-positive cells (early and late apoptotic cells). (d) Caspase-1 activity was measured by a colorimetric assay. Bars with different letters are significantly different from each other $(P<0.05, n=6)$.

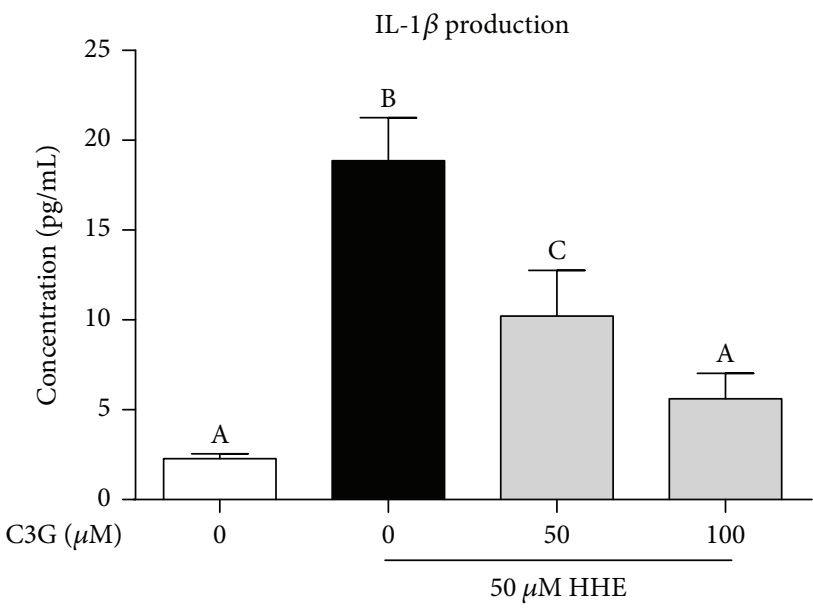

(a)

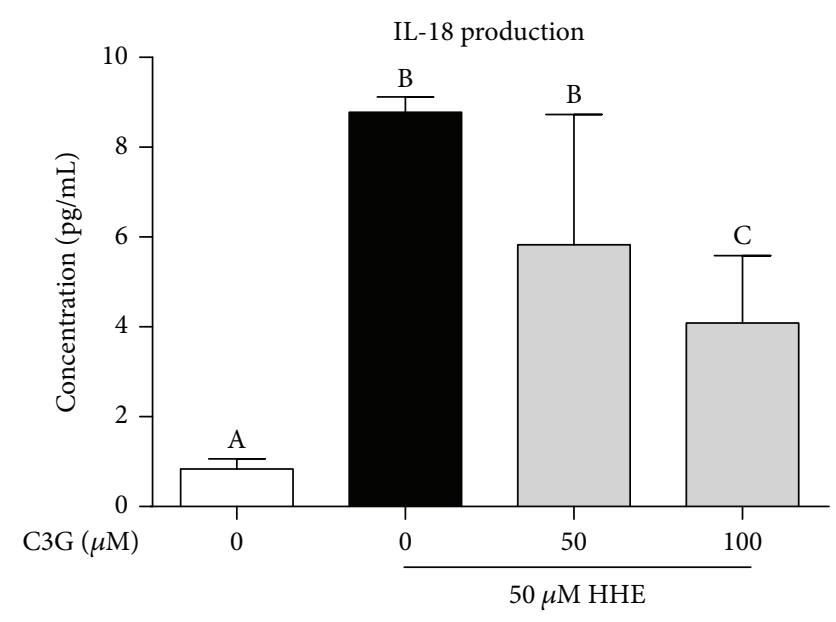

(b)

FIGURE 2: C3G reduced the production of IL- $1 \beta$ and IL-18 induced by HHE in RPE cells. C3Gs (50, $100 \mu \mathrm{M})$ were pretreated to the ARPE-19 cells for $2 \mathrm{~h}$, and then HHE was challenged for $24 \mathrm{~h}$ in serum-free culture mediums. Cell culture mediums were collected, and the production of IL-1 $\beta$ (a) and IL-18 (b) is determined by ELISA. Bars with different letters are significantly different from each other $(P<0.05, n=6)$. 
NLRP3

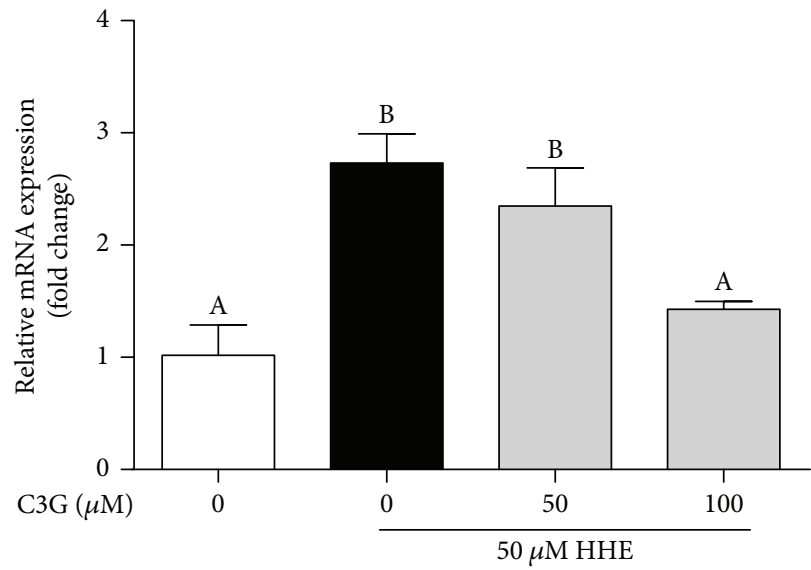

(a)

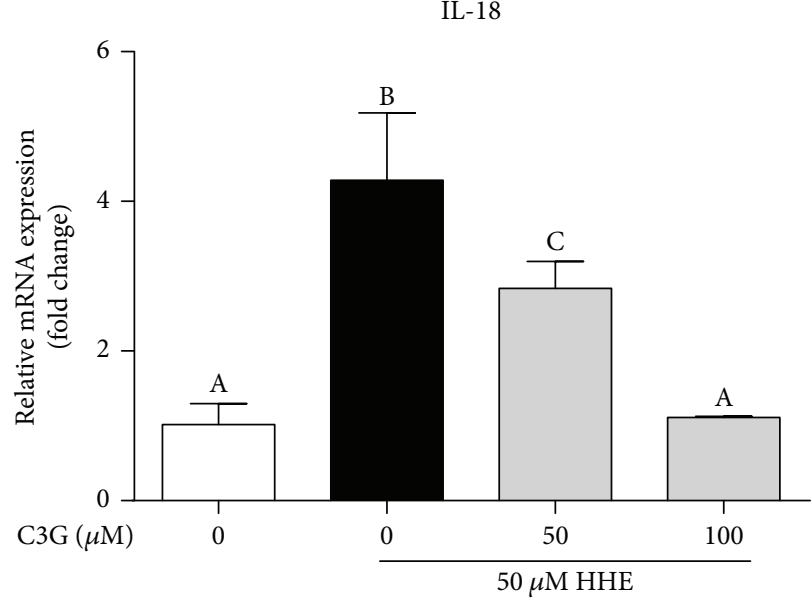

(c)
IL- $1 \beta$

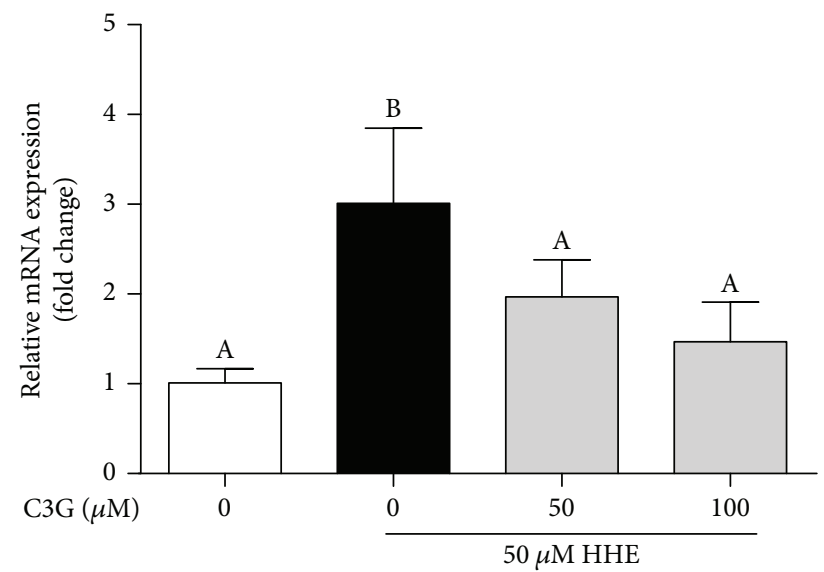

(b)

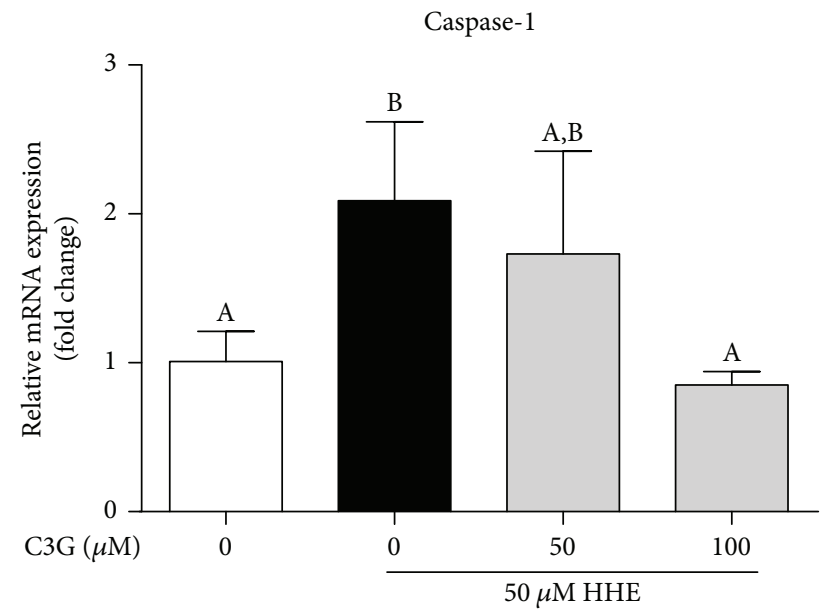

(d)

FIGURE 3: C3G inhibited the activation of NLRP3 inflammasome induced by HHE in RPE cells. C3Gs (50, $100 \mu \mathrm{M})$ were pretreated to the ARPE-19 cells for $2 \mathrm{~h}$, and then HHE was challenged for $6 \mathrm{~h}$ in serum-free culture mediums. Total RNA was collected and relative NLRP3, IL-1 $\beta$, IL-18, and caspase-1 mRNA level are measured by qRT-PCR.

effects on decreasing these gene expressions in HHE-primed ARPE-19 cells (Figure 3(b)-3(d)).

\subsection{JNK Activation Plays a Critical Role during C3G's} Protective Effects against HHE-Induced Cell Death. It was our interest to reveal which signal pathways are involved in the C3G's protective effects to ARPE-19 cells challenged by HHE. ARPE-19 cells were challenged with HHE for various time periods and found that JNK were activated after 30 min treatment of HHE (Figure 4(a)). Indeed, C3G's pretreatment leads to a delay and a decrease on the JNK activation (Figure 4(b)).

3.5. Effects of C3G on AP-1 Activity during HHE-Induced NLRP3 Inflammasome Activation. Accumulating studies suggest that the activation of the transcription factor activator protein $1(\mathrm{AP}-1)$ is essential for the transcriptional regulation of inflammasome-related genes. The effects of AP-1 transcription activity were measured by a dual luciferase reporter gene assay system (Figure 5). The activities of AP-1 transcription factor were significantly increased by HHE and $\mathrm{C} 3 \mathrm{G}$ dose dependently abolished AP-1 transcription activity in ARPE-19 cells.

\section{Discussion}

The lipid peroxidation product 4-hydroxyhexenal (HHE, also known as trans-4-hydroxy-2-hexenal) is an important biochemical mediator originated from n-3 PUFA [14]. Nevertheless, compared with 4-hydroxynonenal (HNE), another major unsaturated aldehyde oxidation product of n-3 PUFA, studies relating to its physiologic/pathological importance are quite limited. Here, we found for the first time that HHE induces cytotoxicity and activates NLRP3 inflammasome in human retinal pigment epithelium cells. Importantly, cyanidin-3-glucoside, a major anthocyanin with great nutritional supplement potentials for preventing retinal degenerative diseases, was able to protect ARPE-19 cells from HHE-induced inflammatory damages. 


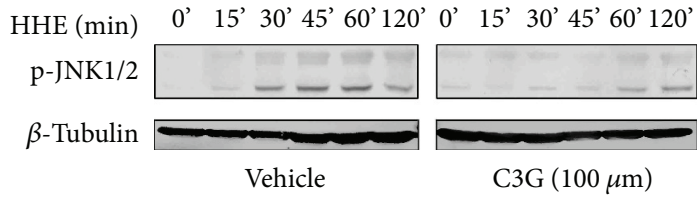

FIGURE 4: C3G blunting HHE-induced JNK activation in RPE cells. Confluent ARPE-19 cells were pretreated with $100 \mu \mathrm{M}$ C3G or vehicle control (DMSO) for $2 \mathrm{~h}$. Then, HHE $(50 \mu \mathrm{M})$ was challenged to the cells for various time periods. Proteins were collected and analyzed using Western blotting.

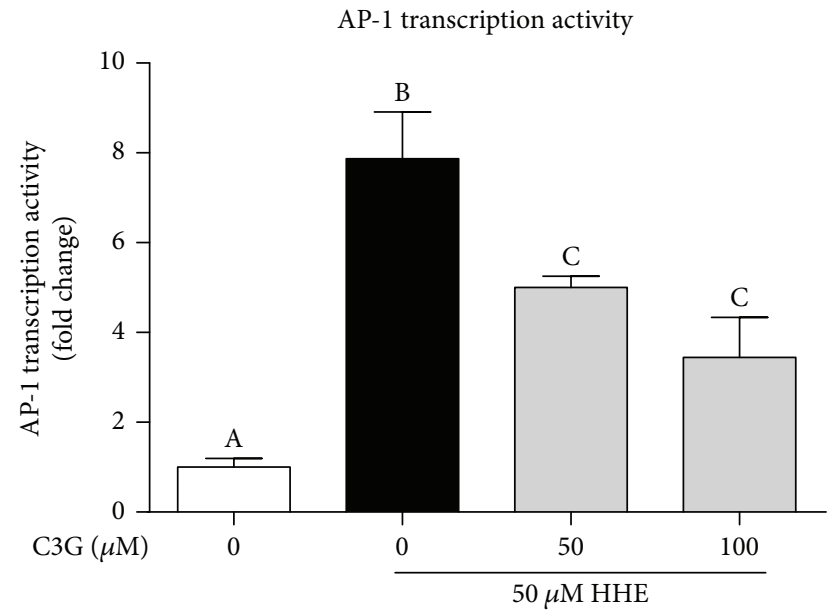

Figure 5: Effects of C3G on AP-1 activity during HHE-induced NLRP3 inflammasome activation. ARPE-19 cells were transfected with plasmid with AP-1 response element (p pAP1-TA-luc) and a Renilla luciferase-expressing vector (pRL-TK) for $24 \mathrm{~h}$. Then, cells were pretreated with $\mathrm{C} 3 \mathrm{G}$ for $2 \mathrm{~h}$ and then $\operatorname{HHE}(50 \mu \mathrm{M})$ was challenged for $12 \mathrm{~h}$ in serum-free culture mediums. The promoter activities of AP-1 were analyzed with a luciferase reporter assay. Values are expressed as the mean \pm SD from three independent experiments. Bars with different letters are significantly different from each other $(P<0.05)$.

During n-3 PUFA peroxidation, several aldehyde products were generated, and at least 15 aldehydes were identified, including HNE, HHE, glyoxal, malondialdehyde, and 2pentenal [14]. Like HNE, comparable cytotoxicity was investigated by researchers in assorted cell types, including primary cells as well as immortalized cell lines. Regarding retinal cells, RPE-19 epithelial cells are more sensitive to toxicity by HNE than HHE, which differs from neuronal system [25]. In our hands, the toxic concentration of HHE $(50 \mu \mathrm{M})$ to RPE-19 cell viability is comparable to previous studies $[26,27]$. In addition, the proapoptotic effects by HHE have also been reported in endothelial cells [28], neuron cells [25], and lens epithelial cells [26, 27]. Despite the activation on caspases of HNE has been noticed by several previous reports, its analog, HHE also showed similar induction effects on caspase-1 $[11,27]$.

It is agreed that caspase- 1 is activated by certain inflammasomes, including NLRP1, NLRP3, NLR family CARDcontaining 4 (NLRC4), and absent in melanoma 2 (AIM2).
Among them, the NLRP3 inflammasome is the best characterized one, which is consisted by a multiprotein complex composed of NLRP3, apoptosis-associated speck-like (ASC) protein, which contains an adaptor for caspase recruitment domain, and procaspase-1 [29]. Selected activators/pathogen stimuli lead to the NLRP3 inflammasome activation, which leads to the molecular modification of procaspase1 , then cleaving it into caspase- 1 and thereby mediating the proinflammatory cytokines, IL- $1 \beta$, and IL-18 maturation and secretion from its precursors. During AMD, a variety of substances leads to the inflammasome activation in the retinal epithelium, including the drusen components [24], the lipofuscin component $N$-retinylidene- $N$-retinylethanolamine (A2E) [3], and the lipid peroxidation product HNE [11]. In our hands, we noticed that HHE treatment also leads to substantial increases of IL- $1 \beta$ and IL18 , which fit well in the classical function of inflammasome pathway.

In vivo and in vitro reports have demonstrated that a dietary supplement of anthocyanin compounds can protect RPE and photoreceptor cells from light and inflammatory damage $[30,31]$. Our previous studies showed the beneficial properties of bilberry-derived polyphenolic compounds (quercetin, anthocyanins, protocatechuic acid, ferulic acid, and chlorogenic acid) against ROS production and inflammatory damages induced by photooxidation in the retina $[18,32]$. C3G is one of the most abundant anthocyanins in the edible parts of plants [18]. Recently, it has gained wide attention for its versatile beneficial effects, like antioxidant [32], antiinflammatory [33], neuroprotective [34], cardiovascular protective properties, and others [35]. In the eyes, it is a strong anthocyanin which alleviated light-induced retinal oxidative stress, inflammation, and apoptosis via activation of Nrf2/HO-1 pathway and inactivation of NF- $\kappa \mathrm{B}$ in pigmented rabbits [18]. Recently, a group of researchers noticed that quercetin protects ARPE-19 cells from HNE-induced cytotoxicity and inflammation [36]. Even though there are no direct studies using HHE-induced ARPE-19 cell inflammatory damage, the anti-inflammatory effects of $\mathrm{C} 3 \mathrm{G}$ are consistent with these studies.

Besides the evidence that showed a drop of HHE-induced NLRP3 inflammasome activation by C3G, we also studied the signaling behind these effects. It has been known that reactive aldehydes, such as HHE and HNE, have been implicated as inducers which activate cellular stress signaling pathways and integrate with additional signals in response for extracellular stimuli [37]. HNE is able to induce both intracellular signaling and intercellular signaling, including NF$\kappa \mathrm{B}, \mathrm{Nrf}-2$ protein kinase $\mathrm{C}$, and MAPK signals. Nevertheless, such studies relating to HHE and cellular signaling are rare compared with HNE. In endothelial cells, HHE was found to induce NF- $\kappa \mathrm{B}$ activation and MAPK activation, which is associated with oxidative stress $[38,39]$. In a model for nonalcoholic fatty liver disease (NAFLD), the persistent JNK activation was noticed by HNE which links to oxidative stress and hepatocyte cell death [40]. During HNE-induced muscle cell apoptosis, HNE leads to the activation of JNK which mediated the antiapoptotic protein inactivation and caspase activation [41]. Pretreatment of well-known antioxidants, 
like resveratrol and piceatannol, suppresses JNK phosphorylation and subsequently blunts the AP-1 signaling $[42,43]$. Given the electrophilic nature of $\alpha, \beta$-unsaturated aldehydes like HNE [14], it is not surprising that HNE activates JNK and AP-1 signaling. Similar to resveratrol, C3G also showed potent regulating effects on these cellular signaling pathways; however, given that inflammasome activation turns on the number of other signaling cascades, the effects of C3G on these cascades need to be clarified in the future.

\section{Conclusion}

Taken together, our study clearly showed that the cytotoxic effects by HHE in APRE-19 cells were significantly rescued by $\mathrm{C} 3 \mathrm{G}$ pretreatment. We also demonstrated for the first time that $\mathrm{C} 3 \mathrm{G}$ inhibited HHE-induced releases of proinflammatory cytokines, IL- $1 \beta$, and IL-18 and blunted NLRP3 inflammasome activation. Moreover, we also showed that JNK-c-Jun/AP-1 pathway activation by HHE is regulated by $\mathrm{C} 3 \mathrm{G}$, which shed a light for future potential treatment against AMD-associated inflammation.

\section{Conflicts of Interest}

The authors declare that they have no conflicts of interest.

\section{Acknowledgments}

The authors gratefully acknowledge the funding support from China Postdoctoral Science Foundation (Grant no. 2017M610133), Beijing Engineering and Technology Research Center of Food Additives, Beijing Technology and Business University (BTBU), and Modern Agro-industry Technology Research System from the Ministry of Agriculture of the People's Republic of China (CARS-22).

\section{References}

[1] A. C. Umfress and M. A. Brantley Jr, "Eye care disparities and health-related consequences in elderly patients with agerelated eye disease," Seminars in Ophthalmology, vol. 31, no. 4, pp. 432-438, 2016.

[2] S. Datta, M. Cano, K. Ebrahimi, L. Wang, and J. T. Handa, "The impact of oxidative stress and inflammation on RPE degeneration in non-neovascular AMD," Progress in Retinal and Eye Research, vol. 60, pp. 201-218, 2017.

[3] C. Brandstetter, F. G. Holz, and T. U. Krohne, "Complement component $\mathrm{C} 5 \mathrm{a}$ primes retinal pigment epithelial cells for inflammasome activation by lipofuscin-mediated photooxidative damage," Journal of Biological Chemistry, vol. 290, no. 52, pp. 31189-31198, 2015.

[4] J. Gao, R. T. Liu, S. Cao et al., "NLRP3 inflammasome: activation and regulation in age-related macular degeneration," Mediators of Inflammation, vol. 2015, Article ID 690243, 11 pages, 2015.

[5] A. Kauppinen, J. J. Paterno, J. Blasiak, A. Salminen, and K. Kaarniranta, "Inflammation and its role in age-related macular degeneration," Cellular and Molecular Life Sciences, vol. 73, no. 9, pp. 1765-1786, 2016.
[6] I. S. Afonina, Z. Zhong, M. Karin, and R. Beyaert, "Limiting inflammation-the negative regulation of NF- $\kappa \mathrm{B}$ and the NLRP3 inflammasome," Nature Immunology, vol. 18, no. 8, pp. 861-869, 2017.

[7] B.-Z. Shao, Z.-Q. Xu, B.-Z. Han, D.-F. Su, and C. Liu, "NLRP3 inflammasome and its inhibitors: a review," Frontiers in Pharmacology, vol. 6, 2015.

[8] A. Abderrazak, T. Syrovets, D. Couchie et al., "NLRP3 inflammasome: from a danger signal sensor to a regulatory node of oxidative stress and inflammatory diseases," Redox Biology, vol. 4, pp. 296-307, 2015.

[9] S. L. Doyle, M. Campbell, E. Ozaki et al., "NLRP3 has a protective role in age-related macular degeneration through the induction of IL-18 by drusen components," Nature Medicine, vol. 18, no. 5, pp. 791-798, 2012.

[10] C. Brandstetter, L. K. M. Mohr, E. Latz, F. G. Holz, and T. U. Krohne, "Light induces NLRP3 inflammasome activation in retinal pigment epithelial cells via lipofuscin-mediated photooxidative damage," Journal of Molecular Medicine, vol. 93, no. 8, pp. 905-916, 2015.

[11] A. Kauppinen, H. Niskanen, T. Suuronen, K. Kinnunen, A. Salminen, and K. Kaarniranta, "Oxidative stress activates NLRP3 inflammasomes in ARPE-19 cells-implications for age-related macular degeneration (AMD)," Immunology Letters, vol. 147, no. 1-2, pp. 29-33, 2012.

[12] R. M. LoPachin and T. Gavin, "Molecular mechanisms of aldehyde toxicity: a chemical perspective," Chemical Research in Toxicology, vol. 27, no. 7, pp. 1081-1091, 2014.

[13] Y. Liu, D. Zhang, J. Hu et al., "Visible light-induced lipid peroxidation of unsaturated fatty acids in the retina and the inhibitory effects of blueberry polyphenols," Journal of Agricultural and Food Chemistry, vol. 63, no. 42, pp. 9295-9305, 2015.

[14] E. K. Long and M. J. Picklo Sr., "Trans-4-hydroxy-2-hexenal, a product of $n-3$ fatty acid peroxidation: make some room HNE," Free Radical Biology and Medicine, vol. 49, no. 1, pp. 1-8, 2010.

[15] Y. Liu, D. Zhang, Y. Wu, and B. Ji, "Docosahexaenoic acid aggravates photooxidative damage in retinal pigment epithelial cells via lipid peroxidation," Journal of Photochemistry and Photobiology B: Biology, vol. 140, pp. 85-93, 2014.

[16] S. M. Yacout and E. R. Gaillard, "The anthocyanins, oenin and callistephin, protect RPE cells against oxidative stress," Photochemistry and Photobiology, vol. 93, no. 2, pp. 590-599, 2017.

[17] S. H. Lee, E. Jeong, S.-S. Paik et al., "Cyanidin-3-glucoside extracted from mulberry fruit can reduce $N$-methyl- $N$-nitrosourea-induced retinal degeneration in rats," Current Eye Research, vol. 39, no. 1, pp. 79-87, 2013.

[18] Y. Wang, Y. Huo, L. Zhao et al., "Cyanidin-3-glucoside and its phenolic acid metabolites attenuate visible light-induced retinal degeneration in vivo via activation of $\mathrm{Nrf2/HO}-1$ pathway and NF- $\kappa$ B suppression," Molecular Nutrition \& Food Research, vol. 60, no. 7, pp. 1564-1577, 2016.

[19] H. Matsumoto, Y. Nakamura, S. Tachibanaki, S. Kawamura, and M. Hirayama, "Stimulatory effect of cyanidin 3glycosides on the regeneration of rhodopsin," Journal of Agricultural and Food Chemistry, vol. 51, no. 12, pp. 3560-3563, 2003.

[20] Y. P. Jang, J. Zhou, K. Nakanishi, and J. R. Sparrow, “Anthocyanins protect against $\mathrm{A} 2 \mathrm{E}$ photooxidation and membrane permeabilization in retinal pigment epithelial cells," Photochemistry and Photobiology, vol. 81, no. 3, pp. 529-536, 2005. 
[21] K. C. Tirupula, F. Balem, N. Yanamala, and J. KleinSeetharaman, "pH-dependent interaction of rhodopsin with cyanidin-3-glucoside. 2. Functional aspects," Photochemistry and Photobiology, vol. 85, no. 2, pp. 463-470, 2009.

[22] Y. Wang, F. Huang, L. Zhao et al., "Protective effect of total flavones from Hippophae rhamnoides L. against visible light-induced retinal degeneration in pigmented rabbits," Journal of Agricultural and Food Chemistry, vol. 64, no. 1, pp. 161-170, 2015.

[23] J. A. Gracie, R. J. Forsey, W. L. Chan et al., "A proinflammatory role for IL-18 in rheumatoid arthritis," The Journal of Clinical Investigation, vol. 104, no. 10, pp. 1393-1401, 1999.

[24] M. Chen, V. O. Ona, M. Li et al., "Minocycline inhibits caspase- 1 and caspase- 3 expression and delays mortality in a transgenic mouse model of Huntington disease," Nature Medicine, vol. 6, no. 7, pp. 797-801, 2000.

[25] E. K. Long, T. C. Murphy, L. J. Leiphon et al., "Trans-4hydroxy-2-hexenal is a neurotoxic product of docosahexaenoic (22:6; n-3) acid oxidation," Journal of Neurochemistry, vol. 105, no. 3, pp. 714-724, 2008.

[26] S. Choudhary, T. Xiao, S. Srivastava et al., "Toxicity and detoxification of lipid-derived aldehydes in cultured retinal pigmented epithelial cells," Toxicology and Applied Pharmacology, vol. 204, no. 2, pp. 122-134, 2005.

[27] S. Choudhary, W. Zhang, F. Zhou et al., "Cellular lipid peroxidation end-products induce apoptosis in human lens epithelial cells," Free Radical Biology and Medicine, vol. 32, no. 4, pp. 360-369, 2002.

[28] J. Y. Lee, J. H. Je, D. H. Kim et al., "Induction of endothelial apoptosis by 4-hydroxyhexenal," European Journal of Biochemistry, vol. 271, no. 7, pp. 1339-1347, 2004.

[29] J.-J. Jhang and G.-C. Yen, "The role of Nrf2 in NLRP3 inflammasome activation," Cellular \& Molecular Immunology, vol. 14, no. 12, pp. 1011-1012, 2017.

[30] Y. Liu, X. Song, D. Zhang et al., "Blueberry anthocyanins: protection against ageing and light-induced damage in retinal pigment epithelial cells," British Journal of Nutrition, vol. 108, no. 1, pp. 16-27, 2011.

[31] Y. Wang, D. Zhang, Y. Liu, D. Wang, J. Liu, and B. Ji, “The protective effects of berry-derived anthocyanins against visible light-induced damage in human retinal pigment epithelial cells," Journal of the Science of Food and Agriculture, vol. 95, no. 5, pp. 936-944, 2015.

[32] Y. Wang, H. J. Kim, and J. R. Sparrow, "Quercetin and cyanidin-3-glucoside protect against photooxidation and photodegradation of A2E in retinal pigment epithelial cells," Experimental Eye Research, vol. 160, pp. 45-55, 2017.

[33] D. Serra, L. M. Almeida, and T. C. P. Dinis, “Anti-inflammatory protection afforded by cyanidin-3-glucoside and resveratrol in human intestinal cells via Nrf2 and PPAR- $\gamma$ : comparison with 5-aminosalicylic acid," Chemico-Biological Interactions, vol. 260, pp. 102-109, 2016.

[34] A. Tarozzi, F. Morroni, A. Merlicco et al., "Neuroprotective effects of cyanidin 3-O-glucopyranoside on amyloid beta (25-35) oligomer-induced toxicity," Neuroscience Letters, vol. 473, no. 2, pp. 72-76, 2010.

[35] P. Raj, J. L. McCallum, C. Kirby et al., "Effects of cyanidin 3-0glucoside on cardiac structure and function in an animal model of myocardial infarction," Food \& Function, vol. 8, no. 11, pp. 4089-4099, 2017.
[36] M. Hytti, N. Piippo, A. Salminen, P. Honkakoski, K. Kaarniranta, and A. Kauppinen, "Quercetin alleviates 4hydroxynonenal-induced cytotoxicity and inflammation in ARPE-19 cells," Experimental Eye Research, vol. 132, pp. 208-215, 2015.

[37] S. S. Singhal, S. P. Singh, P. Singhal, D. Horne, J. Singhal, and S. Awasthi, "Antioxidant role of glutathione S-transferases: 4-hydroxynonenal, a key molecule in stress-mediated signaling," Toxicology and Applied Pharmacology, vol. 289, no. 3, pp. 361-370, 2015.

[38] J. H. Je, J. Y. Lee, K. J. Jung et al., "NF- $\kappa$ B activation mechanism of 4-hydroxyhexenal via NIK/IKK and p38 MAPK pathway," FEBS Letters, vol. 566, no. 1-3, pp. 183-189, 2004.

[39] J. Y. Lee, J. H. Je, K. J. Jung, B. P. Yu, and H. Y. Chung, "Induction of endothelial iNOS by 4-hydroxyhexenal through NF- $\kappa \mathrm{B}$ activation," Free Radical Biology and Medicine, vol. 37, no. 4, pp. 539-548, 2004.

[40] R. Singh, Y. Wang, J. M. Schattenberg, Y. Xiang, and M. J. Czaja, "Chronic oxidative stress sensitizes hepatocytes to death from 4-hydroxynonenal by JNK/c-Jun overactivation," American Journal of Physiology-Gastrointestinal and Liver Physiology, vol. 297, no. 5, pp. G907-G917, 2009.

[41] M. Braga, A. P. Sinha Hikim, S. Datta et al., "Involvement of oxidative stress and caspase 2-mediated intrinsic pathway signaling in age-related increase in muscle cell apoptosis in mice," Apoptosis, vol. 13, no. 6, pp. 822-832, 2008.

[42] O. Kutuk, G. Poli, and H. Basaga, "Resveratrol protects against 4-hydroxynonenal-induced apoptosis by blocking JNK and c-JUN/AP-1 signaling," Toxicological Sciences, vol. 90, no. 1, pp. 120-132, 2006.

[43] Y. J. Jang, J. E. Kim, N. J. Kang, K. W. Lee, and H. J. Lee, “Piceatannol attenuates 4-hydroxynonenal-induced apoptosis of PC12 cells by blocking activation of c-Jun N-terminal kinase," Annals of the New York Academy of Sciences, vol. 1171, no. 1, pp. 176-182, 2009. 


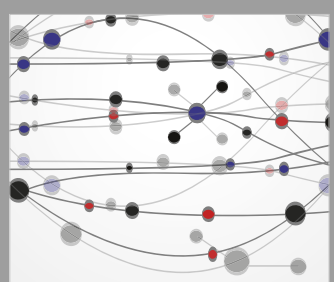

The Scientific World Journal
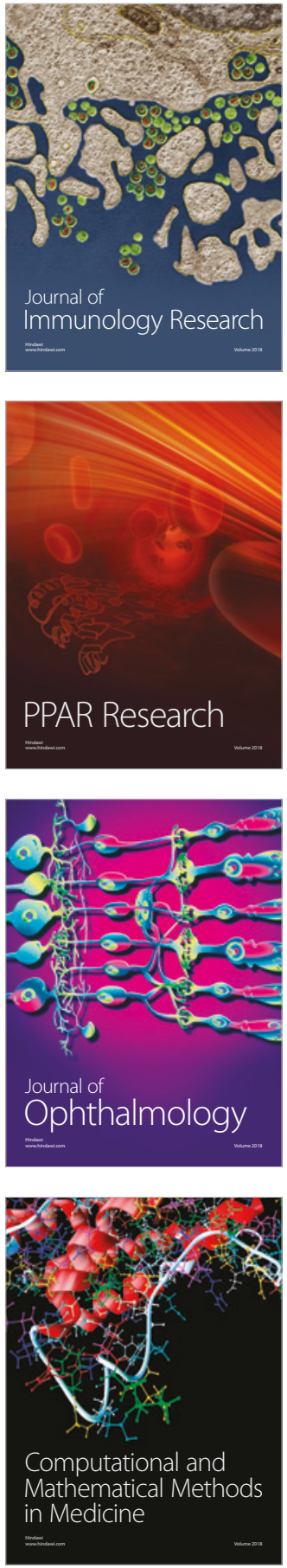

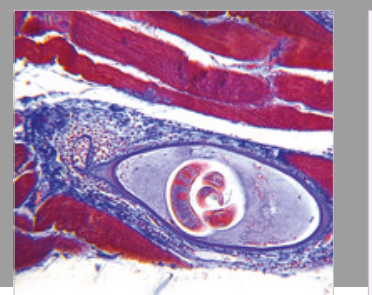

Gastroenterology Research and Practice

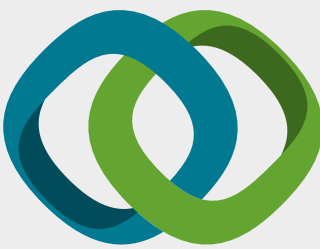

\section{Hindawi}

Submit your manuscripts at

www.hindawi.com
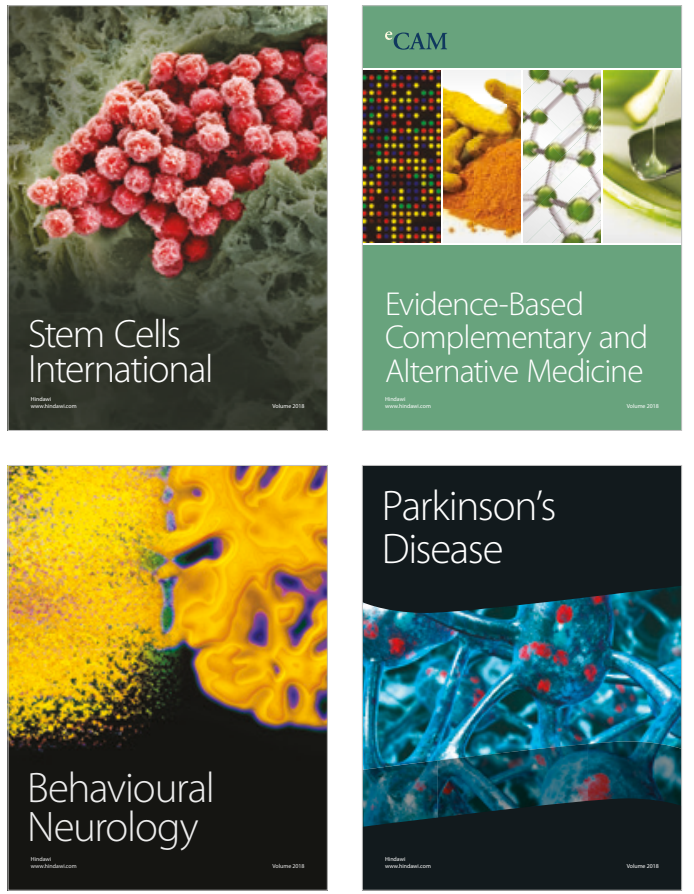

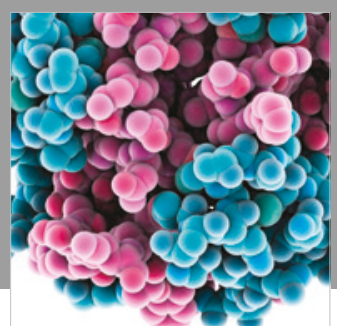

ournal of

Diabetes Research

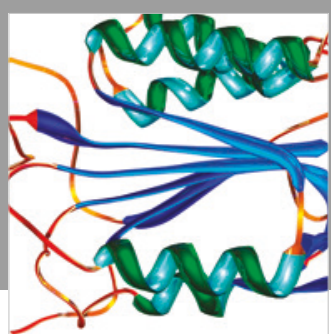

Disease Markers
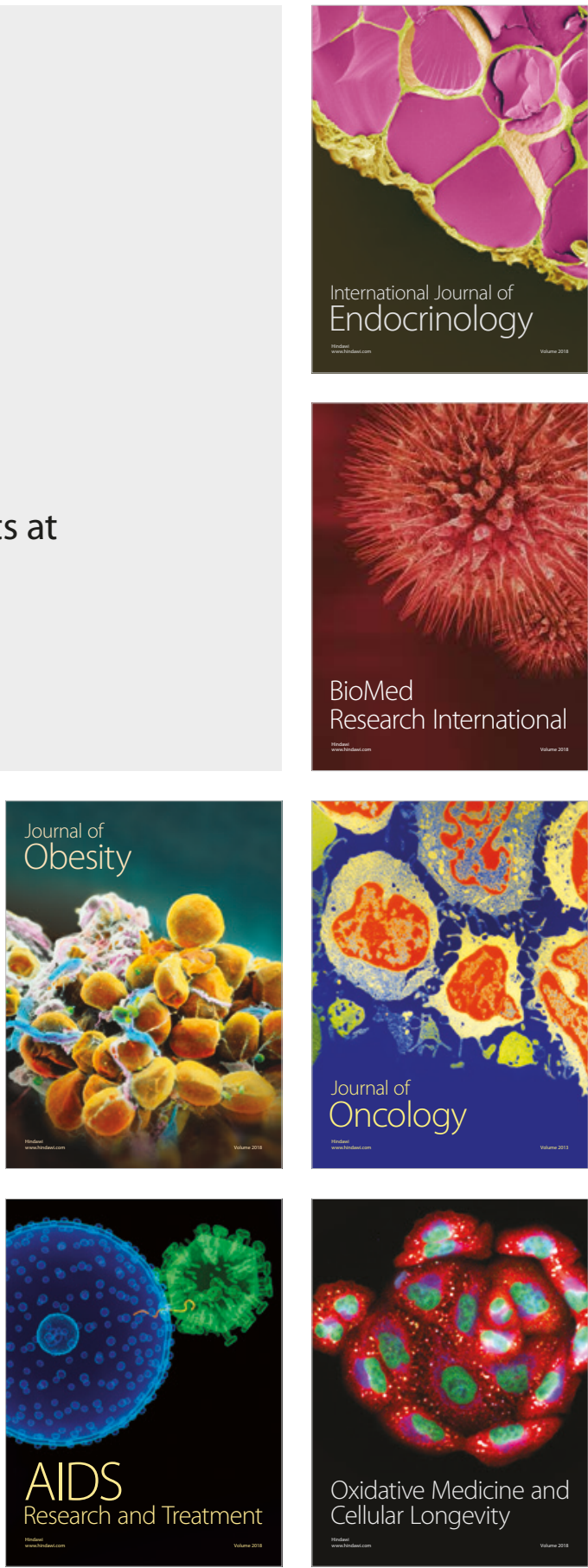